\title{
Neon and oxygen in low activity stars: towards a coronal unification with the Sun
}

\author{
J. Robrade ${ }^{1}$, J. H. M. M. Schmitt ${ }^{1}$, and F. Favata ${ }^{2}$ \\ 1 Universität Hamburg, Hamburger Sternwarte, Gojenbergsweg 112, 21029 Hamburg, Germany \\ e-mail: jrobrade@hs .uni-hamburg.de \\ 2 ESA - Planning and Community Coordination Office, 8-10 rue Mario Nikis, 75738 Paris Cedex 15, France
}

Received 29 February 2008 / Accepted 27 May 2008

\section{ABSTRACT}

\begin{abstract}
Aims. The disagreement between helioseismology and a recent downward revision of solar abundances has resulted in a controversy about the true neon abundance of the Sun and other stars. We study the coronal Ne/O abundance ratios of nearby stars with modest activity levels and investigate a possible peculiarity of the Sun among the stellar population in the solar neighborhood.

Methods. We used XMM-Newton and Chandra data from a sample of weakly and moderately active stars with $\log L_{\mathrm{X}} / L_{\mathrm{bol}} \approx-5 \ldots-7$ to investigate high-resolution X-ray spectra to determine their coronal $\mathrm{Ne} / \mathrm{O}$ abundance ratio. We applied two linear combinations of strong emission lines from neon and oxygen, as well as a global-fitting method for each dataset, and crosschecked the derived results. Results. The sample stars show a correlation between their Ne/O ratio and stellar activity in the sense that stars with a higher activity level show a higher $\mathrm{Ne} / \mathrm{O}$ ratio. We find that the $\mathrm{Ne} / \mathrm{O}$ abundance ratio decreases in our sample from values of $\mathrm{Ne} / \mathrm{O} \approx 0.4$ down to $\mathrm{Ne} / \mathrm{O} \approx 0.2-0.25$, suggesting that ratios similar to "classical" solar values, i.e. $\mathrm{Ne} / \mathrm{O} \approx 0.2$, are rather common for low activity stars. A significantly enhanced neon abundance as the solution to the solar modeling problem seems unlikely.

Conclusions. From the coronal Ne/O abundance ratios, we find no indications of a peculiar position of the Sun among other stars. The solar behavior appears to be rather typical of low activity stars.
\end{abstract}

Key words. stars: abundances - stars: activity - stars: coronae - X-rays: stars

\section{Introduction}

The chemical composition of the Sun is used as a reference frame throughout astronomy and therefore the precise determination of the solar abundances is of particular interest. Beside indirect methods utilizing solar wind particles and meteorites, the measurement of photospheric lines is used as the prime method to derive the chemical composition of the Sun. Making use of more and more refined measurements and modeling, the chemical composition of the Sun "evolved" throughout the decades, however abundance compilations like the ones of Anders \& Grevesse (1989) and more recently by Grevesse \& Sauval (1998) have become widely accepted. Asplund et al. (2005) proposed the most recent revision of the solar abundance scale based on a new $3 \mathrm{D}$ hydrodynamic modeling of the solar atmosphere, resulting in approximately $30 \%$ lower abundances of many light elements including in particular $\mathrm{C}, \mathrm{N}, \mathrm{O}$ and Ne. While this new solar chemical composition provides a better agreement with e.g. measurements of the local ISM, on the solar side severe discrepancies appeared (Basu \& Antia 2004; Turck-Chièze et al. 2004; Bahcall et al. 2005a). The light elements are an important source of opacity in the Sun and as a consequence the almost perfect agreement between helioseismology and models of the solar interior based on the abundance values of Grevesse \& Sauval (1998) is seriously disturbed. The disagreement is far beyond measurement errors and estimated modeling uncertainties and therefore a solution of this problem is highly desired.

One proposal to reconcile the new abundances with helioseismology suggests an enhancement of the solar neon abundance (Antia \& Basu 2005). In contrast to C, N, O, Ne has no strong photospheric lines and its abundance is determined from emission lines originating in the transition region and the corona or through composition measurements of solar wind particles. However, both methods do not necessarily trace the true solar photospheric abundances, since chemical fractionation processes in the outer atmospheric layers of stars may be present. For the Sun the FIP (First Ionization Potential) effect is known, leading to an overabundance of low FIP $(\$ 10 \mathrm{eV})$ elements like $\mathrm{Fe}$ or $\mathrm{Mg}$, whereas the high FIP elements $\mathrm{Ne}$ and $\mathrm{O}$ (that is used as reference element) are thought to remain around photospheric values (see e.g. Geiss 1998).

While the "classical" solar Ne/O ratio from Grevesse \& Sauval (1998) is $\mathrm{Ne} / \mathrm{O}=0.18$, the overall lower Asplund et al. (2005) abundances result in a comparable value of $\mathrm{Ne} / \mathrm{O}=0.15$. However, the Asplund et al. (2005) abundances require a significant increase of the solar neon abundance by, depending on the assumed model and abundance uncertainties, a factor of $\sim 2.5$ up to 4.0 (Antia \& Basu 2005; Bahcall et al. 2005b), i.e. $\mathrm{Ne} / \mathrm{O} \approx 0.4-0.6$ could provide the missing opacity and would reconcile helioseismology with the new abundances derived by Asplund et al. (2005). Evidence for an increased neon abundance was presented by Drake \& Testa (2005), who measured coronal $\mathrm{Ne} / \mathrm{O}$ ratios in X-ray spectra of various nearby stars, found a value of $\mathrm{Ne} / \mathrm{O}=0.41$ and "solved" the problem by assuming a similar ratio for the Sun, thought noting that solar measurements indicate the lower values, i.e. $\mathrm{Ne} / \mathrm{O}=0.15 / 0.18$ to be correct. Actually their new neon abundance is still slightly below the required enhancement, but assuming other elemental abundances 
to additionally increase jointly within allowed errors would provide a sufficient opacity increase. Several objections to this solution were raised, especially from solar observers. A reassessment of solar coronal data from the Solar Maximum Mission led to an upper limit of $\mathrm{Ne} / \mathrm{O}=0.18 \pm 0.04$ for active regions (Schmelz et al. 2005) and an analysis of transition region lines observed by the $\mathrm{SOHO}$ satellite suggested $\mathrm{Ne} / \mathrm{O}=0.17 \pm 0.05$ for the quiet Sun (Young 2005), both confirming the "classical" solar $\mathrm{Ne} / \mathrm{O}$ ratio. Although sometimes higher $\mathrm{Ne} / \mathrm{O}$ values were determined from X-ray spectra of energetic flares or in $\gamma$-ray production regions (see e.g. Table 1, sup. data of Drake \& Testa 2005), solar measurements are overall consistent with a ratio of $\mathrm{Ne} / \mathrm{O} \approx 0.2 \pm 0.05$, as also noticed in Drake \& Testa (2005). Furthermore, the stellar sample used by Drake \& Testa (2005) for their abundance analysis contains mostly active stars and binary systems, which are not comparable to the Sun in terms of activity and are thought to exhibit a different chemical fractionation process. This so-called inverse FIP effect (see Güdel 2004, for overall X-ray properties of stellar coronae) is commonly observed in X-ray spectra of active stars. It is possible that the inverse FIP effect modifies photospheric $\mathrm{Ne} / \mathrm{O}$ ratios, leading to higher coronal values than in the photosphere. Hence it is necessary to determine the $\mathrm{Ne} / \mathrm{O}$ ratio in relatively inactive stars that are comparable to the Sun. Since low activity stars are intrinsically X-ray faint, only a few nearby stars have been investigated and their $\mathrm{Ne} / \mathrm{O}$ ratios were mostly found to be below the Drake \& Testa (2005) value but higher than the "classical" solar one, although measurement errors for individual stars were often quite large.

Two different methods have been used to investigate stellar coronal abundance ratios in general; on one hand, by global spectral modeling that usually includes the determination of key elemental abundances and some kind of emission measure distribution (EMD), on the other hand, by using individual or a combination of emission lines that have a similar emissivity vs. temperature distributions and thus the determined ratio is almost independent of the stellar EMD and the temperature dependence cancels out. This latter method requires adequate and sufficiently strong lines for both elements in question, here $\mathrm{Ne}$ and $\mathrm{O}$. Already Acton et al. (1975) pointed out that the emissivities of the O VIII Ly $\alpha$ and the Ne IX resonance line have a similar temperature dependence and are therefore suitable to determine the solar $\mathrm{Ne} / \mathrm{O}$ ratio, which they determined to $\mathrm{Ne} / \mathrm{O}=0.21 \pm 0.07$. This method has been refined by using linear combinations of suited lines to minimize temperature dependent residuals, specifically Drake \& Testa (2005) included the Ne X Ly $\alpha$ line and Liefke \& Schmitt (2006) also the O VII resonance line. In general, inclusion of more lines, especially from different ionization stages, should minimize the temperature dependence, but adds measurement errors and restricts the method to detectors that spectrally resolve and cover all involved lines with sufficient sensitivity.

In this work we present a study of low to medium activity stars with $\log L_{\mathrm{X}} / L_{\mathrm{bol}} \lesssim-5$ and spectral types mid-F to mid-K, for which high resolution X-ray spectra are available. All stars in our sample are main-sequence stars or only moderately evolved, i.e. they belong to luminosity classes IV-V. We specifically determine coronal $\mathrm{Ne} / \mathrm{O}$ abundance ratios for these stars by using two linear combinations of $\mathrm{Ne}$ and $\mathrm{O}$ lines as well as global spectral fits and check the results obtained from the different methods among each other and against available literature values. We further compare our results to those obtained for more active stars and investigate a possible abundance peculiarity of the Sun.
Our paper is structured as follows. In Sect. 2 we describe the applied methods and data used, in Sect. 3 we present our results, check the applied methods and discuss implication of our findings in the context of solar and stellar coronal abundances and finally in Sect. 4 we summarize our findings and conclusions.

\section{Observations and data analysis}

Our sample contains low to medium activity stars with spectral types mid-F to mid-K, for which high-resolution X-ray spectra are available. In the next subsections we first describe the stellar sample and the observations, followed by a detailed description of the data analysis.

\subsection{Targets and observations}

Our stellar sample consists of those stars from the XMM-Newton monitoring program on coronal activity cycles, i.e. $61 \mathrm{Cyg}$ (K5+K7) (Hempelmann et al. 2006), $\alpha$ Cen (G2+K1) (Robrade et al. 2005, 2007) and HD 81809 (G2+G9) (Favata et al. 2004), as well as the stars $\beta$ Com (G0), $\epsilon$ Eri (K2) and Procyon (F5). The targets $61 \mathrm{Cyg}, \alpha$ Cen and HD 81809 have been observed regularly for several years by XMM-Newton in short exposures separated half a year each. These observations have resulted in roughly ten available exposures for each target, however for the faintest target HD 81809 we used only exposures taken during its X-ray brighter phases. The high resolution spectra of HD 81809 and $61 \mathrm{Cyg}$ are presented here for the first time and we reexamine $\alpha$ Cen using a more extended dataset and analysis compared to Liefke \& Schmitt (2006). The components of the $\alpha$ Cen system undergo strong, probably cyclic variability (Robrade et al. 2005, 2007), therefore the contributions of the individual stars to the flux of the system heavily depends on the phase of the observations. This system is considered a prime test case since $\alpha$ Cen A is a close-by, nearly perfect solar twin and therefore should exhibit very similar properties.

We further (re-)analyzed and re-examined XMM-Newton data of $\beta$ Com, $\epsilon$ Eri and Procyon and Chandra/LETGS data of $\alpha$ Cen, $\epsilon$ Eri and Procyon, which we retrieved from the archives. In the context of stellar global coronal properties, the data of Procyon has been (partly) presented by Raassen et al. (2002) and Sanz-Forcada et al. (2004), $\beta$ Com is included in the "Sun in time" sample (Telleschi et al. 2005), the LETGS spectra of $\alpha$ Cen in Raassen et al. (2003) and $\epsilon$ Eri in Wood \& Linsky (2006). All observations used in this analysis are summarized in Table 1. We consider our dataset to constitute an overall representive of these stars and include phases of activity common for these objects. Spatially resolved high resolution X-ray spectra of the binary systems are only available in the $\alpha$ Cen/Chandra case, all other data represent the summed flux of both components of the respective system. The used XMM-Newton data were taken with the EPIC (European Photon Imaging Camera) MOS detectors and the RGS (Reflection Grating Spectrometer, 5-38 ), all analyzed Chandra observations used the LETGS (Low Energy Transmission Grating Spectrometer, 2-170 A) together with the HRC-S.

\subsection{Data analysis}

The XMM-Newton data analysis was carried out with the Science Analysis System (SAS) version 7.0 (Loiseau et al. 2006). Standard selection criteria were applied to the data and periods with enhanced background due to proton flares were 
Table 1. Observing log of the X-ray data used and effective exposure time of the filtered high resolution data.

\begin{tabular}{lllll}
\hline \hline Star & Mission & Year(s) & No Exp. & Obs.(ks) \\
\hline 61 Cyg & XMM & $2002-2007$ & 11 & 103 \\
$\alpha$ Cen & XMM & $2003-2007$ & 9 & 73 \\
$\alpha$ Cen & Chandra & 1999 & 1 & 79 \\
$\beta$ Com & XMM & 2003 & 1 & 41 \\
$\epsilon$ Eri & XMM & 2003 & 1 & 13 \\
$\epsilon$ Eri & Chandra & 2001 & 1 & 105 \\
HD 81809 & XMM & $2001-03,2006-07$ & 7 & 72 \\
Procyon & XMM & 2000,2007 & 3 & 138 \\
Procyon & Chandra & 1999 & 2 & 139 \\
\hline
\end{tabular}

discarded. To increase the signal-to-noise-ratio of the RGS data we further restricted the time intervals with acceptable background rates (CCD9 rate $<0.5 \mathrm{cts} / \mathrm{s}$ ) for fainter sources like $\beta$ Com and HD 81809 and merged the individual observations for each targets using the tool "rgscombine". The Chandra data was analyzed with standard tools of CIAO 3.4 (Fruscione et al. 2006), we applied standard data processing and filtering and we used positive and negative first order spectra to measure line fluxes. The individual LETGS exposures provide sufficient data quality and are analyzed separately. To investigate global stellar X-ray properties, we use a combination of RGS/MOS data from the XMM-Newton observations, for Chandra LETGS we use spectra in the 10-100 $\mathrm{A}$ range. For the determination of the $\mathrm{Ne} / \mathrm{O}$ ratios only high resolution X-ray spectra with good SNR are taken into account.

Global spectral analysis was performed with XSPEC V11.3 (Arnaud 1996) using multi-temperature models (three temperature components are sufficient to describe our spectra) with variable abundances as calculated with the APEC code (Smith et al. 2001). In our models, temperatures, emission measure and abundances of $\mathrm{Ne}, \mathrm{O}$ and $\mathrm{Fe}$ are treated as free parameters. Depending on spectral quality, the abundances of other elements were also free parameters or set to solar values. We also checked the results by fitting only those spectral ranges dominated by $\mathrm{Ne}$ and $\mathrm{O}$ lines, i.e. $11.0-14.0 \AA, 18.0-23.0 \AA$, and additionally 85.0-100.0 $\AA$ for the LETGS. We find that while the absolute abundances of $\mathrm{Ne}$ and $\mathrm{O}$ differ somewhat between the applied models, mainly due to the interdependence with the emission measure, the derived $\mathrm{Ne} / \mathrm{O}$ ratio turns out to be a quite stable result.

For line fitting purposes we use the CORA program (Ness \& Wichmann 2002), using identical line width and assuming Lorentzian line shapes for all lines of a respective detector. This analysis uses total spectra, i.e. we determine one background (+continuum) level in the respective region around each line. To derive the $\mathrm{Ne} / \mathrm{O}$ ratios from linear combinations of emission lines we especially measure the O VIII Ly $\alpha$ line $(18.97 \AA$ ) and the O VII resonance line $(21.6 \AA)$ as well as the Ne X Ly $\alpha$ line $(12.13 \AA)$ and the Ne IX resonance line (13.45 $\AA$ ). Note that both Ly $\alpha$ lines are actually unresolved doublets and that the strength of the resonance lines of $\mathrm{O}$ VII and Ne IX were derived from a fit to the respective triplet with all three lines included in the fit. As an example, we show in Fig. 1 the X-ray spectrum of $61 \mathrm{Cyg}$ from the RGS detectors with the spectral lines of interest labeled. The spectrum consists of 11 co-added observations, background subtraction and flux conversion were done with the "rgsfluxer" tool.

In the line ratio approach one uses linear combinations of these line fluxes to determine the $\mathrm{Ne} / \mathrm{O}$ abundance ratio,

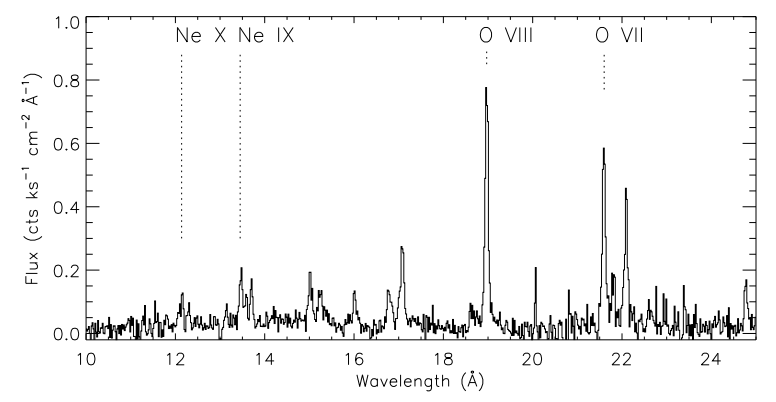

Fig. 1. High resolution X-ray spectrum of $61 \mathrm{Cyg}$ obtained with the RGS detectors; used spectral lines of neon and oxygen are labeled.

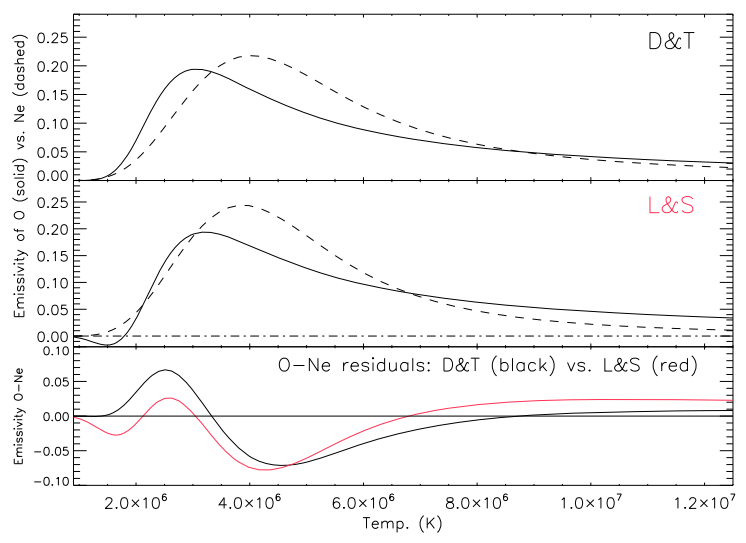

Fig. 2. Theoretical emissivity curves for neon and oxygen as used by Drake \& Testa (2005) (upper panel) and Liefke \& Schmitt (2006) (middle panel) and respective residuals (lower panel) vs. temperature. Note that D\&T used erg $\mathrm{cm}^{3} \mathrm{~s}^{-1}$, while L\&S used photons $\mathrm{cm}^{3} \mathrm{~s}^{-1}$, both in arbitrary units (see text).

however, the specific linear combination can be differently chosen. In Fig. 2 we show the emissivity curves, i.e. $G(T)$, of the respective linear combinations for $\mathrm{Ne}$ and $\mathrm{O}$ as used by Drake \& Testa (2005) (D\&T) and Liefke \& Schmitt (2006) (L\&S) and calculated with the CHIANTI V 5.2 code (Dere et al. 1997; Landi et al. 2006). Unfortunately the original weighting is based on energy flux in D\&T (O VIII vs. Ne IX $+0.15 \times \mathrm{Ne} \mathrm{X})$ and on photon flux in L\&S $(0.67 \times \mathrm{O}$ VIII- $0.17 \times \mathrm{O}$ VII vs. Ne IX $+0.02 \times \mathrm{Ne} \mathrm{X})$, we therefore normalized the distributions for comparison. Note that the L\&S emissivity for $\mathrm{O}$ is unphysically negative at temperatures below 1.8 MK, corresponding to an O VIII Ly $\alpha / \mathrm{O}$ VII(r) energy flux ratio of $\sim 0.3$ or lower. As is apparent from Fig. 2, for both combinations the respective $\mathrm{Ne}$ and $\mathrm{O}$ curves are quite similar, but the residuals average out only over a very broad temperature range. It is important to keep in mind, that the temperature dependence of the residuals result in systematic errors of the derived $\mathrm{Ne} / \mathrm{O}$ ratios, which need not to be small in disadvantageous cases, e.g. for weakly active stars. In this case the exact value strongly depends on the used linear combination and the underlying stellar EMD. In order to illustrate this behavior, we show in Fig. 3 the summed residuals of the O-Ne emissivities scaled with the summed, average emissivity vs. temperature; i.e.

$$
\sum_{T=0}^{T_{\max }} \frac{G_{\mathrm{Ne}}(T)-G_{\mathrm{O}}(T)}{\frac{1}{2}\left(G_{\mathrm{Ne}}(T)+G_{\mathrm{O}}(T)\right)}
$$

physically corresponding to the simplified case of cool star with a flat EMD over the respective temperature range. Neglecting very low temperatures where values are far outside the plotting range, the summed residuals from D\&T are positive 


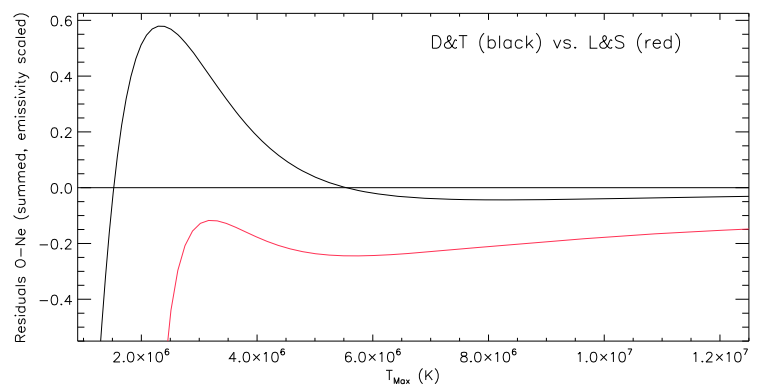

Fig. 3. Summed and scaled residuals of the emissivities $(\mathrm{O}-\mathrm{Ne})$ for the linear combinations used by D\&T and L\&S. Positive values correspond to an overprediction of oxygen, negative ones to an overprediction of neon (see text).

below $~ 5 \mathrm{MK}$ and become slightly negative for higher temperatures, the L\&S values are negative for all temperatures. In summary, the residuals in these emissivity ratios smooth out in stars with a sufficiently broad temperature distribution as noted by Drake \& Testa (2005); however, this is not the case for stars with only cool or very dominant cool coronal plasma.

Here we investigate medium and low activity stars, whose coronal plasma temperatures are usually below $10 \mathrm{MK}$ and our sample stars are, as shown in Sect. 3, clearly dominated by relatively cool plasma below $5 \mathrm{MK}$ during these observations. In the spectra of weakly active stars $\mathrm{Ne} \mathrm{X}$ with a peak formation temperature of $\approx 6 \mathrm{MK}$ is often virtually absent, further its contribution to the emissivity of neon in the used linear combinations is secondary. This makes the precise measurement of the Ne IX resonance line a crucial point in this analysis that contributes greatly to the overall uncertainties. Further, contrary to the $\mathrm{O}$ VII and $\mathrm{O}$ VIII lines, which are quite isolated in the X-ray spectra, Ne X and Ne IX are found in a spectral region where several blends with Fe lines are present, which may influence the accurate determination of the neon abundance. Since there are systematic, temperature dependent errors in the linear combinations, we crosscheck these results with those from the multitemperature models described above, which are based on a different analysis method and use a different data base.

\section{Results}

Our sample stars differ in spectral type and level of activity as expressed by the $L_{X} / L_{\text {bol }}$ ratio, therefore we first present global properties and give measured line strengths relevant for our study, while the derived $\mathrm{Ne} / \mathrm{O}$ ratios and a discussion of the results in the solar context will be presented in the following subsections.

\subsection{Global stellar properties}

We first determined the global X-ray properties of our sample stars from RGS and MOS spectra and present in Table 2 the respective X-ray luminosity, mean temperature and activity level together with basic stellar parameters adopted from the Simbad database. For cyclic stars and unresolved binaries the X-ray data represent the average state of activity during the analyzed observations. Bolometric luminosities were calculated from the apparent visual magnitudes, the determined values of $L_{\mathrm{bol}}$ indicate that especially HD 81809 and Procyon, which has luminosity class IV-V, are somewhat evolved and already above the main sequence.
Table 2. Basic parameters of our sample stars. $L_{\mathrm{X}}(0.2-3.0 \mathrm{keV})$ and $\mathrm{X}$-ray flux ratios from $\mathrm{RGS} / \mathrm{MOS}$ data.

\begin{tabular}{lllllll}
\hline \hline Star & Type & $\begin{array}{l}V \\
(\mathrm{mag})\end{array}$ & $\begin{array}{l}\text { Dist. } \\
(\mathrm{pc})\end{array}$ & $\begin{array}{l}\log L_{\mathrm{X}} \\
(\mathrm{erg} / \mathrm{s})\end{array}$ & $\begin{array}{l}T_{\mathrm{Av}} \\
(\mathrm{MK})\end{array}$ & $\begin{array}{l}L_{\mathrm{X}} / L_{\mathrm{bol}} \\
\log \end{array}$ \\
\hline 61 Cyg & $\mathrm{K} 5+\mathrm{K} 7$ & $5.2+6.0$ & 3.5 & 27.3 & 3.2 & $-5.6,-5.5$ \\
$\alpha$ Cen & $\mathrm{G} 2+\mathrm{K} 1$ & $0.0+1.3$ & 1.3 & 27.1 & 2.2 & $-7.3,-6.2$ \\
$\beta$ Com & $\mathrm{G} 0$ & 4.26 & 9.2 & 28.2 & 3.4 & -5.6 \\
$\epsilon$ Eri & $\mathrm{K} 2$ & 3.73 & 3.2 & 28.2 & 3.8 & -4.9 \\
HD 81809 & $(\mathrm{G} 2+\mathrm{G} 9)^{*}$ & $5.8+6.8$ & 31.2 & 28.7 & 4.0 & -5.6 \\
Procyon & $\mathrm{F} 5+(\mathrm{WD})$ & 0.34 & 3.5 & 27.9 & 1.9 & -6.5 \\
\hline
\end{tabular}

* Unresolved in X-rays.

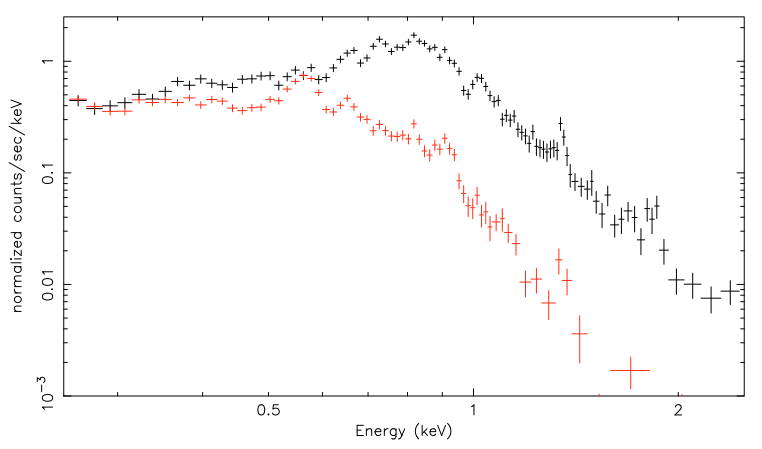

Fig. 4. X-ray spectra of $\epsilon$ Eri (black) and Procyon (red) obtained with the $\operatorname{MOS}$ detector $(1 \mathrm{keV} \hat{=} 12.4 \AA)$.

In Fig. 4 we show the MOS spectra of the moderately active star $\epsilon$ Eri and the weakly active stars Procyon. The star $\epsilon$ Eri exhibits a considerably harder spectrum compared to Procyon, indicating a significantly hotter corona. We quote X-ray luminosities in the $0.2-3.0 \mathrm{keV}$ band, i.e. where the MOS instruments provided useful data. The corresponding $L_{\mathrm{X}}$ in the ROSAT band $(0.1-2.4 \mathrm{keV})$ is, depending on the spectral shape, roughly 20-40\% higher for our sample stars. The binary systems $61 \mathrm{Cyg}$ and $\alpha$ Cen are spatially resolved in the X-ray images obtained by the MOS detectors and we give the average activity level of each component separately. Both respective components are identified as variable X-ray emitters (Robrade et al. 2007), with $\alpha$ Cen B (K1) and 61 Cyg A (K5) being the X-ray dominant components during our observations. HD 81809 is unresolved in all $\mathrm{X}$-ray detectors and thus the main contributor to its X-ray emission is unknown.

The derived average coronal temperatures of $2-4 \mathrm{MK}$ are typical for weakly to moderately active stars and their low X-ray luminosities, or alternatively $L_{\mathrm{X}} / L_{\mathrm{bol}}$, confirms this finding. While our sample stars span about two orders of magnitude in activity, really active stars have $\log L_{\mathrm{X}} / L_{\mathrm{bol}} \approx-3$ and are therefore a hundred times more active than our most active star. The temperature of HD 81809 appears quite high given its $L_{\mathrm{X}} / L_{\text {bol }}$ ratio; this is mainly due to an observation taken during its brightest phase when the source was also hot, however without exhibiting clear flare signatures (see discussion in Favata et al. 2004). In our global analysis we also investigated the abundances of other key elements and we find indications of an enhancement of low FIP elements $(\mathrm{Fe}, \mathrm{Mg})$, resembling the solar FIP effect, in some of the less active stars like $\beta$ Com and most clearly in $\alpha$ Cen. In contrast, the FIP effect is rather weak or even not present in the moderately active stars like $61 \mathrm{Cyg}$ and $\epsilon$ Eri, but also in the more evolved low-activity star Procyon. 
Table 3. Measured line fluxes in $10^{-5}$ photons $\mathrm{cm}^{-2} \mathrm{~s}^{-1}$ from RGS or LETGS $(\mathrm{C})$ and energy flux ratio of the $\mathrm{O}$ VIII $(\mathrm{Ly} \alpha)$ to $\mathrm{O}$ VII $(\mathrm{r})$ line.

\begin{tabular}{lllll|l}
\hline \hline Star & O VII $(r)$ & O VIII & Ne IX $(r)$ & Ne X $^{*}$ & O8/O7 $(r)$ \\
\hline 61 Cyg & $6.6 \pm 0.4$ & $9.1 \pm 0.5$ & $2.0 \pm 0.3$ & $1.6 \pm 0.2$ & $1.57 \pm 0.13$ \\
$\alpha$ Cen & $33.5 \pm 1.3$ & $27.0 \pm 1.2$ & $4.1 \pm 0.5$ & $1.6 \pm 0.2$ & $0.92 \pm 0.05$ \\
$\alpha$ Cen 03/04 & $47.9 \pm 2.1$ & $45.4 \pm 1.5$ & $5.5 \pm 0.8$ & $2.4 \pm 0.5$ & $1.08 \pm 0.06$ \\
$\alpha$ Cen A (C) & $9.2 \pm 1.0$ & $3.2 \pm 0.5$ & $0.5 \pm 0.3$ & $\lessgtr 0.2$ & $0.40 \pm 0.07$ \\
$\alpha$ Cen B (C) & $11.5 \pm 1.0$ & $6.3 \pm 0.6$ & $1.3 \pm 0.4$ & $\lesssim 0.2$ & $0.62 \pm 0.08$ \\
$\beta$ Com & $3.8 \pm 0.6$ & $5.9 \pm 0.6$ & $0.9 \pm 0.3$ & $0.6 \pm 0.3$ & $1.77 \pm 0.33$ \\
$\epsilon$ Eri & $44.1 \pm 2.6$ & $78.0 \pm 3.0$ & $21.2 \pm 2.4$ & $10.5 \pm 1.4$ & $2.01 \pm 0.14$ \\
$\epsilon$ Eri (C) & $41.5 \pm 1.6$ & $78.9 \pm 1.7$ & $18.8 \pm 0.9$ & $16.7 \pm 0.9$ & $2.16 \pm 0.10$ \\
HD 81809 & $1.0 \pm 0.3$ & $2.3 \pm 0.3$ & $0.5 \pm 0.2$ & $0.5 \pm 0.2$ & $2.62 \pm 0.86$ \\
Procyon & $35.6 \pm 1.1$ & $22.9 \pm 0.9$ & $1.8 \pm 0.3$ & $0.5 \pm 0.2$ & $0.73 \pm 0.04$ \\
Procyon (C) & $29.1 \pm 1.6$ & $17.3 \pm 1.0$ & $2.5 \pm 0.5$ & $0.7 \pm 0.3$ & $0.68 \pm 0.05$ \\
Procyon (C) & $30.3 \pm 1.7$ & $18.9 \pm 1.1$ & $1.4 \pm 0.3$ & $\lessgtr 0.2$ & $0.71 \pm 0.06$ \\
\hline
\end{tabular}

* Blended with Fe XVII.

\subsection{Neon and oxygen line fluxes}

We measured photon fluxes of the spectral lines of interest as described above from the respective high resolution spectra of our targets and listed their values in Table 3. For the $\alpha$ Cen system we also investigated the data from the years 2003/04 separately to search for possible changes in its $\mathrm{Ne} / \mathrm{O}$ ratio. During these years $\alpha$ Cen $\mathrm{A}$ was in an X-ray bright phase and contributed most strongly to the allover X-ray emission, on the other hand the overall activity level of the system was high. We also derive the $\mathrm{O}$ VIII $(\mathrm{Ly} \alpha) / \mathrm{O} \mathrm{VII}(\mathrm{r})$ energy flux ratio for each dataset, a ratio that is a good temperature proxy for the cooler X-ray emitting plasma and most sensitive in the $1.5-5 \mathrm{MK}$ temperature range. This ratio allows to crosscheck the global fitting results and is further used to investigate if the Chandra observations are performed at a comparable activity level for our targets. We find that the O VIII/O VII ratio correlates very well with the average temperature derived from the global spectral fitting of the MOS/RGS data.

Comparing the XMM-Newton and Chandra measurements obtained for the same stars, we find nearly identical activity levels for Procyon and a slightly hotter but still comparable state in the LETGS data for $\epsilon$ Eri. Completely different values are found for $\alpha$ Cen, which was apparently in a far cooler and X-ray fainter state during the Chandra observation, even when compared to the averaged RGS value from all XMM-Newton observations. In comparison with the LETGS analysis by Raassen et al. (2003), we find similar values for the individual line fluxes, however our derived X-ray luminosities are significantly lower by a factor of $\sim 3$. We find X-ray luminosities of roughly $3 \times 10^{26} \mathrm{erg} / \mathrm{s}$ for $\alpha$ Cen A and $4 \times 10^{26} \mathrm{erg} / \mathrm{s}$ for $\alpha$ Cen B, i.e. $\log L_{\mathrm{X}}=26.8 \mathrm{erg} / \mathrm{s}$ for the total system in the $0.2-3.0 \mathrm{keV}$ band, i.e. three times fainter than the derived $L_{X}$ from the XMM-Newton measurements, in line with the low fluxes measured in the emission lines and supported by the low $\mathrm{O}$ VIII $(\operatorname{Ly} \alpha) / \mathrm{O}$ VII(r) ratio. In Procyon we find an apparent discrepancy by a factor of roughly two in the strength of the Ne IX and NeX lines between the AO-0 (indicating higher fluxes) and AO-1 observations from Chandra/LETGS, although statistically only at the $2 \sigma$ level. Since the RGS flux is intermediate between these measurements and the Ne VII and Ne VIII lines around $88.1 \AA$ are comparable in both LETGS observations, this might be a chance effect.
Table 4. Derived Ne/O ratio for various methods (sorted by decreasing average activity).

\begin{tabular}{lccc}
\hline \hline Star & D\&T & L\&S & Global fit \\
\hline$\epsilon$ Eri (RGS) & $0.41 \pm 0.05$ & $0.46 \pm 0.06$ & $0.36 \pm 0.04$ \\
$\epsilon$ Eri (LETGS) & $0.38 \pm 0.02$ & $0.40 \pm 0.02$ & $0.35 \pm 0.04$ \\
61 Cyg & $0.34 \pm 0.05$ & $0.39 \pm 0.06$ & $0.36 \pm 0.05$ \\
HD 81809 & $0.36 \pm 0.15$ & $0.35 \pm 0.13$ & $0.36 \pm 0.22$ \\
$\beta$ Com (RGS) & $0.24 \pm 0.08$ & $0.27 \pm 0.09$ & $0.25 \pm 0.13$ \\
$\alpha$ Cen B & $0.29 \pm 0.07$ & $0.54 \pm 0.16$ & $0.26 \pm 0.16$ \\
$\alpha$ Cen (RGS) & $0.23 \pm 0.03$ & $0.32 \pm 0.04$ & $0.25 \pm 0.03$ \\
$\alpha$ Cen (03/04-RGS) & $0.18 \pm 0.03$ & $0.24 \pm 0.04$ & $0.24 \pm 0.04$ \\
Procyon (RGS) & $0.12 \pm 0.02$ & $0.19 \pm 0.03$ & $0.21 \pm 0.03$ \\
Procyon (LETGS0) & $0.21 \pm 0.04$ & $0.36 \pm 0.08$ & $0.23 \pm 0.03$ \\
Procyon (LETGS1) & $0.10 \pm 0.03$ & $0.18 \pm 0.04$ & $0.21 \pm 0.03$ \\
$\alpha$ Cen A & $0.24 \pm 0.12$ & $0.85 \pm 0.61$ & $0.19 \pm 0.18$ \\
\hline
\end{tabular}

\subsection{Stellar $\mathrm{Ne} / \mathrm{O}$ abundance ratios}

In order to obtain the true flux of the $\mathrm{Ne}$ and $\mathrm{O}$ lines, we have to take possible line blends into account. We identify relevant blends from atomic line lists, we inspected Chianti V5.2 and SPEX V2.0, and deblended the lines when necessary with theoretical emissivities to ensure a uniform analysis. While no significant blends are present for the used $\mathrm{O}$ lines, the $\mathrm{NeX}$ line is blended with Fe XVII at $12.12 \AA$, with both ions having similar peak formation temperatures of $T_{\mathrm{Max}} \approx 5.5 \mathrm{MK}$. Inspecting the respective line emissivities and assuming a solar abundance pattern the contamination of the NeX line is estimated to be around $20 \%$. The contamination is higher by a factor of up to three in those stars that show a strong FIP effect. Fortunately these stars tend to have intrinsically cool coronae and $\mathrm{Ne} \mathrm{X}$ is mostly weak or even undetected, allowing this simplified approach. The Ne IX triplet with $T_{\mathrm{Max}}=3.9 \mathrm{MK}$ is again blended with several Fe lines, however the dominant blends are from Fe XIX $\left(T_{\text {Max }}=8.1 \mathrm{MK}\right)$ with its strongest line being located at $13.52 \AA$. Even in our "hottest" sample stars coronal plasma with temperatures around $8 \mathrm{MK}$ contributes only little to the overall coronal emission and further this blend mainly affects the intercombination line at $13.55 \AA$, which is not used in this analysis. We therefore expect an essentially negligible Fe contamination of the Ne IX resonance line for our sample stars and use its measured flux, whereas we reduce the measured Nex flux by $20 \%$. The stellar Ne/O ratios from the linear combinations were then derived using these values and are given in Table 4 together with the ratios from global spectral modeling. The quoted errors are statistical errors due to spectral fitting and measurements of $\mathrm{Ne}$ and $\mathrm{O}$ lines only and they do not take into account any systematic errors.

Inspecting the results of the $\mathrm{Ne} / \mathrm{O}$ ratios derived from the different methods, we find a quite good overall agreement for most sample stars. Very similar values of the $\mathrm{Ne} / \mathrm{O}$ ratio derived from the three methods are generally obtained for those stars whose underlying EMD covers a sufficiently broad range of temperatures, provided that a well exposed spectrum is available. However, this is hardly achieved by inactive stars and only the more active stars in our sample roughly fulfill this criterion. The most obvious discrepancies can thus be attributed to either systematic errors, that are expected from the theoretical calculations presented in Sect.2, or to cases where statistical errors are large. There appears to be a tendency to an overprediction of $\mathrm{Ne}$ in the L\&S model for all sample stars and of O in the D\&T model for the lowest activity stars, exactly reflecting the theoretical expectations and hence pointing to the presence of systematic 


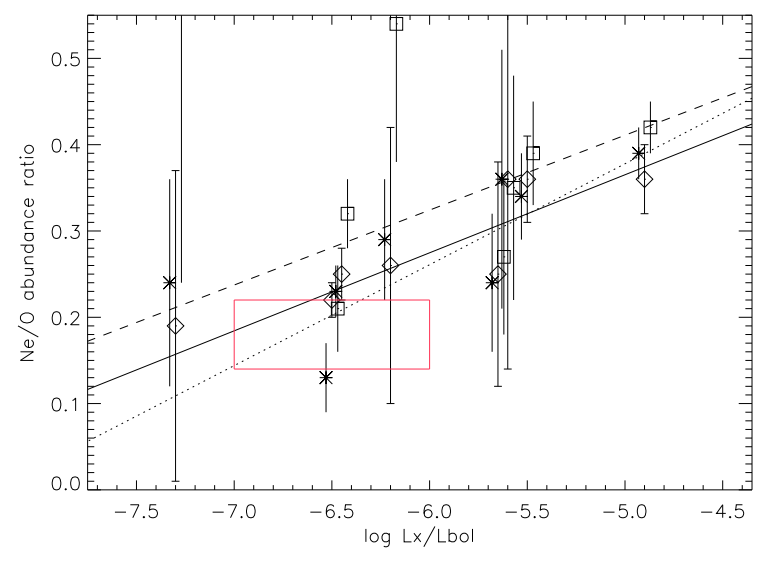

Fig. 5. Ne/O ratios of our sample stars derived with var. methods (global fit: diamonds/solid line; D\&T: asterisks/dotted line; L\&S: squares/dashed line) and the "classical" Sun (red box).

errors. However, measurement errors and limited statistics prevent a more detailed comparison. Therefore in many cases the true ratio should be in-between the results from two linear combinations and thus together they define a reasonable range of allowed values. The $\mathrm{Ne} / \mathrm{O}$ abundances derived from our global fits indeed result mostly in intermediate values when compared to those from the line ratios, this is also represented by an intermediate regression curve shown in Fig. 5. In summary, both methods generally complement among each another, however the results derived from linear combinations of emission lines have to be treated with some caution when considering individual targets with unknown underlying EMD. Nevertheless, all methods indicate a clear correlation between $\mathrm{Ne} / \mathrm{O}$ ratio and coronal activity.

In Fig. 5 we show the Ne/O ratio vs. stellar activity for our sample stars and the Sun as derived from the three applied methods; slight offsets in $\log L_{\mathrm{X}} / L_{\mathrm{bol}}$ among the methods are introduced for clarity. Our measurements clearly show a trend of lower $\mathrm{Ne} / \mathrm{O}$ ratio towards less active stars, a finding that is independent of the applied method. We also plotted the error weighted linear regression for each method, whereas for stars with multiple observations the error weighted mean of the individual results is used. Given our lack of any definite physical model, the assumed linear dependence is the simplest possible fit over the considered parameter range. Although more complicated dependencies obviously cannot be ruled out, a general decline of the $\mathrm{Ne} / \mathrm{O}$ abundance with activity is present in all three regression curves. Admittedly some of the results for individual stars have large statistical errors and our stellar sample is quite limited, however our results clearly suggest low $\mathrm{Ne} / \mathrm{O}$ ratios for the weakly active stars. Also, the overplotted range of "classical" solar values for activity and $\mathrm{Ne} / \mathrm{O}$ ratio fits well into the respective parameter space derived from our low-activity stars.

Our results also agree reasonably well with available literature values (transformed to Grevesse \& Sauval 1998 abundance scale) derived from various multi-temperature EMD modeling, despite different applied methods in data analysis, underlying atomic data, data sets or selection criteria. Concerning the individual targets, the most active star in our sample, the K-dwarf $\epsilon$ Eri, shows the highest ratio with $\mathrm{Ne} / \mathrm{O}=0.35-0.4$. A similar range of values is present in the literature, e.g. $\mathrm{Ne} / \mathrm{O}=0.36$ (Wood \& Linsky 2006) or Ne/O = 0.4 (Sanz-Forcada et al. 2004). For the group of slightly less active stars we measure $\mathrm{Ne} / \mathrm{O} \approx 0.35$. The stars $61 \mathrm{Cyg}, \beta \mathrm{Com}$ and HD 81809 have very similar activity levels and there might to be a trend towards lower $\mathrm{Ne} / \mathrm{O}$ ratios for stars of earlier spectral type at a given activity level. Especially the $\mathrm{G} 0$-star $\beta$ Com is better described by a ratio around $\mathrm{Ne} / \mathrm{O}=0.25$, but given the errors and the small sample size of only three stars, this has to be checked with larger and better observed stellar samples. For the less active $\alpha$ Cen system we find values around $\mathrm{Ne} / \mathrm{O}=0.25$. Additionally, in an analysis of selected phases of the $\alpha$ Cen data where the contribution of the lesser active component $\alpha$ Cen A to the RGS spectra is maximal, indications of a decrease in the $\mathrm{Ne} / \mathrm{O}$ ratio of the $\alpha$ Cen system are present. This points to a lower $\mathrm{Ne} / \mathrm{O}$ ratio in $\alpha$ Cen A compared to $\alpha$ Cen B. Raassen et al. (2003) derived values of $\mathrm{Ne} / \mathrm{O}=0.18 \pm 0.07(\mathrm{~A})$ and $0.24 \pm 0.09(\mathrm{~B})$ for the individual components of the $\alpha$ Cen system from the LETGS data, slightly lower than our values, but showing the same trend in the $\mathrm{Ne} / \mathrm{O}$ ratio of the components as our results for the phase selected data. Also, the $\mathrm{Ne} / \mathrm{O}=0.27$ result from their line based analysis in Liefke \& Schmitt (2006) fits into this picture, when keeping in mind that they used the RGS data of the $\alpha$ Cen system from 2003-2005, i.e. intermediate in terms of activity and contributing components between our 2003/04 dataset and the full data selection. However, given the measurement errors this has to be confirmed by further observations. Procyon, the least active star in our sample beside $\alpha$ Cen A, again shows a very low $\mathrm{Ne} / \mathrm{O}$ ratio for most of the data used. While Sanz-Forcada et al. (2004) derived $\mathrm{Ne} / \mathrm{O}=0.4$ from the LETGS data (AO0+AO1), Raassen et al. (2002) derived a value of $\mathrm{Ne} / \mathrm{O}=0.22$ for the RGS as well as the LETGS data (AO1). Some of these discrepancies are possibly due to the different analyzed datasets as discussed above. Although the even lower $\mathrm{Ne} / \mathrm{O}$ ratios derived from the linear combination of D\&T probably have to be at least partially attributed to systematic errors, values around $\mathrm{Ne} / \mathrm{O}=0.2$ are found repeatedly in various global analysis and linear combination models for Procyon. A value of $\mathrm{Ne} / \mathrm{O}=0.2$ also corresponds to the mean from all used methods and datasets, whereas our averaged global analysis yields $\mathrm{Ne} / \mathrm{O}=0.22$ for Procyon.

In summary, for moderately active $\mathrm{G}$ - and K-dwarfs with activity levels of $\log L_{\mathrm{X}} / L_{\text {bol }} \approx-5 \ldots-5.5$ we measure abundance ratios of $\mathrm{Ne} / \mathrm{O}=0.3-0.4$. While the $\mathrm{Ne} / \mathrm{O}$ ratio further decreases with decreasing stellar activity, values around $\mathrm{Ne} / \mathrm{O}=$ $0.2-0.25$ are found exclusively in the least active stars with activity levels around and below $\log L_{\mathrm{X}} / L_{\text {bol }} \approx-6.5$. Additionally, none of these low activity stars shows a significantly higher ratio, therefore low $\mathrm{Ne} / \mathrm{O}$ ratios seem to be common for stars with activity levels comparable to the Sun. This finding is also supported by phase selected and spatially resolved analysis of the $\alpha$ Cen system, however measurement errors become large for these data. A possible further dependence of spectral type, activity phase or other stellar parameters like luminosity class, binarity etc. might be present, but cannot be investigated with the present data.

We finally discuss the observed change of the $\mathrm{Ne} / \mathrm{O}$ ratio in the context of more active stars. The ratio increases from $\mathrm{Ne} / \mathrm{O} \approx 0.2$ up to $\mathrm{Ne} / \mathrm{O} \approx 0.4$ when going from low to moderately activity stars, i.e. from $\log L_{\mathrm{X}} / L_{\text {bol }} \approx-7$ to $\log L_{\mathrm{X}} / L_{\text {bol }} \approx$ -5 . Given these finding it is not surprising that Drake $\&$ Testa (2005) found an average ratio of $\mathrm{Ne} / \mathrm{O}=0.41$ for a stellar sample dominated by stars or binary systems that are mostly ten to hundred times more active than the stars in our sample. If anything, in the light of the inferred trend for the $\mathrm{Ne} / \mathrm{O}$ ratio, their value appears rather low than high. This further points to a different behavior when going to more extreme activity levels. While a linear dependence between $\mathrm{Ne} / \mathrm{O}$ ratio and $\log L_{\mathrm{X}} / L_{\mathrm{bol}}$ is a fair description for our stellar sample, we already noted that other models would also fit the data. Taking their active main sequence stars and binary systems into account, we suspect the $\mathrm{Ne} / \mathrm{O}$ ratio 
to saturate already at values around $\mathrm{Ne} / \mathrm{O} \approx 0.4-0.5$. However, the detailed investigation of such a flattening or saturation of the $\mathrm{Ne} / \mathrm{O}$ ratio towards very active stars is beyond the scope of this study, but deserves further attention.

\subsection{Discussion in the solar context}

When putting the derived stellar $\mathrm{Ne} / \mathrm{O}$ ratios in the context with the Sun, we first have to consider the solar activity level and the well known variations of the Sun's X-ray emission over the course of the activity cycle. Further, most of the solar data refer to individual regions and the usually spatially resolved solar data has to be transformed into some kind of point-source like data that is available for stars. Using Yohkoh/SXT measurement taken from 1991-1995 and covering roughly a solar half cycle from maximum to minimum, Acton (1996) derived a conversion factor between the observed X-ray index and the solar energy flux in the energy range $0.2-4.5 \mathrm{keV}$, leading to a solar activity range of $\log L_{\mathrm{X}} / L_{\mathrm{bol}} \approx-7.9 \ldots-6.5$ and average coronal temperatures between 2-3 MK. Another approach based on Yohkoh/SXT data made by Peres et al. (2000) resulted in a solar activity range over the cycle of $\log L_{\mathrm{X}} / L_{\mathrm{bol}} \approx-7.2 \ldots-6.0$, while Judge et al. (2003) derived a slightly more active Sun with an activity range of $\log L_{\mathrm{X}} / L_{\mathrm{bol}} \approx-6.9 \ldots-5.8$ from SNOE/SXP data. These literature values were transformed to our $0.2-3.0 \mathrm{keV}$ band by a $20 \%$ reduction of the given $0.1-2.4 \mathrm{keV}$ flux. Note that even larger discrepancies by up to an order of magnitude are present between literature values for the Sun's X-ray output (see e.g. Table 2 in Judge et al. 2003), thus complicating a comparison with our sample stars. Averaged over recent measurements we find a solar X-ray luminosity range of $4 \times 10^{26}-4 \times 10^{27} \mathrm{erg} \mathrm{s}^{-1}$, i.e. an activity range over the cycle of $\log L_{\mathrm{X}} / L_{\mathrm{bol}} \approx-7 \ldots-6$. Hence the average X-ray activity level of the Sun appears to be comparably to those of the $\alpha$ Cen system and Procyon, while the Sun is significantly less active than the rest of the sample stars. Given the above derived correlation between activity and $\mathrm{Ne} / \mathrm{O}$ ratio, only these stars are considered further in our discussion on $\mathrm{Ne} / \mathrm{O}$ ratios at solar-like activity levels.

Our low activity stars show ratios around $\mathrm{Ne} / \mathrm{O} \approx 0.2-0.25$, i.e. close to the "classical" solar ratio of $\mathrm{Ne} / \mathrm{O}=0.18 \pm 0.04$ (Grevesse \& Sauval 1998). This value is consistent with emission line measurements for the supergranule cells/network, representing the quiet Sun (Young 2005), as well as for active regions (Schmelz et al. 2005), therefore the Ne/O ratio seems to be in general rather independent of the analyzed solar structure when overall properties are considered. Spatially and temporally restricted analysis of X-ray and $\gamma$-ray lines from individual flares indicate a more diverse picture for local phenomena; they either point to an enhancement of low FIP elements (McKenzie \& Feldman 1992), i.e. lower $\mathrm{Ne} / \mathrm{O}$ ratios, a rather constant $\mathrm{Ne} / \mathrm{O}$ ratio (Widing \& Feldman 1995) or to an enhanced ratio in the range of $\mathrm{Ne} / \mathrm{O} \approx 0.25$ (Ramaty et al. 1995) up to $\mathrm{Ne} / \mathrm{O} \approx 0.3$ (Schmelz 1993). While these events may not be overall representive of the atmospheric layers discussed here, the chemical fractionation also seems to depend on the particular properties of the observed event. Further, fairly constant $\mathrm{Ne}$ and $\mathrm{O}$ abundances were also found in the composition of the solar wind or flare particles obtained by various instruments (Geiss 1998), again referring to a rather average solar property. Therefore all present evidence points to "unified" abundance ratio of $\mathrm{Ne} / \mathrm{O} \approx 0.2 \pm 0.05$ in the outer atmospheric layers of the Sun and other weakly active stars. We note, that $\mathrm{Ne} / \mathrm{O}=0.15$ from the revised abundances of Asplund et al. (2005) does not fit equally well, but is also consistent with the stellar data given the present uncertainties. However, in this case the solar interior problem remains.

A significantly higher neon abundance around $\mathrm{Ne} / \mathrm{O} \approx 0.5$ as proposed for reconciliation between helioseismology and the revised abundances can be ruled out for the coronae of weakly active stars, consistent with the findings for the Sun's outer layers. A higher photospheric neon abundance is of course not in conflict with our data, however such a scenario would require some unknown but universal fractionation process that essentially decouples the photospheric neon abundance from the coronal one in the Sun and other low activity stars without significantly influencing oxygen at the same time. This hypothesized mechanism would have to operate, despite both elements being high FIP elements, have similar properties and ionization times in solar fractionation modeling, exhibit a roughly constant ratio in various solar surface features of different activity level or show similar properties in solar wind data (von Steiger \& Geiss 1989; Geiss 1998). We note that chemical fractionation models for different solar layers and structures are still under debate (e.g. McKenzie et al. 1998), however we feel that such a scenario seems to be quite unlikely and suppose that further revised solar modeling will settle the matter.

Thus we cannot resolve the discrepancy between recent modeling of solar abundances and helioseismology with our stellar Ne/O abundance measurements and no other element than neon is easily at hand to provide the missing opacity, due to the fact that the abundances of other important elements can be constrained by photospheric measurements. However, at least solar coronal properties appear to fit very well into the X-ray picture derived for low activity stars with similar spectral type and evolutionary phase. Therefore the Sun seems to be in no way exceptional or peculiar and behaves just like a typical, weakly active, middle aged star.

\section{Summary and conclusions}

1. We have determined the coronal $\mathrm{Ne} / \mathrm{O}$ abundance ratio in a sample of low and moderately active stars. For stars in the activity range $\log L_{\mathrm{X}} / L_{\text {bol }} \approx-5 \ldots-7$ we find a trend of decreasing $\mathrm{Ne} / \mathrm{O}$ ratio with decreasing activity level. The $\mathrm{Ne} / \mathrm{O}$ ratio decreases from values of $\mathrm{Ne} / \mathrm{O} \approx 0.4$ for moderately active stars down to $\mathrm{Ne} / \mathrm{O} \approx 0.2-0.25$ for low activity stars. The decrease is measured independently of the chosen analysis method, i.e. by global spectral modeling and two linear combinations of strong emission lines.

2. The Sun as a low activity star fits very well into the picture derived from the stellar X-ray data. A low Ne/O ratio around the "classical" solar value of $\mathrm{Ne} / \mathrm{O}=0.18 \mathrm{might}$ be rather typical for solar-like stars with comparable activity level. A significant higher neon abundance, i.e. a ratio of $\mathrm{Ne} / \mathrm{O}=0.4-0.6$, as proposed to solve the solar interior problem can be virtually ruled out for the coronal composition. It also appears unlikely for the photospheric composition given the present knowledge and observational data on chemical fractionation processes in weakly active stars.

3. We consider the main findings of this study to be robust, however the presently available data is insufficient to determine the detailed characteristics of the decline. While a linear dependence between $\mathrm{Ne} / \mathrm{O}$ ratio and activity level describes our data well, some kind of flattening or saturation is expected for very active stars. Possible dependences of abundance fractionation on other stellar parameters beside activity also remain unclear. However, further X-ray observations 
of nearby low activity stars may successfully address these problems and tighten their re-unification with the Sun.

Acknowledgements. This work is based on observations obtained with $X M M-N e w t o n$, an ESA science mission with instruments and contributions directly funded by ESA Member States and NASA and it has made use of data obtained from the Chandra Data Archive. J.R. acknowledges support from DLR under 50OR0105.

\section{References}

Acton, L. 1996, in Cool Stars, Stellar Systems, and the Sun, ed. R. Pallavicini, \& A. K. Dupree, ASP Conf. Ser., 109, 45

Acton, L. W., Catura, R. C., \& Joki, E. G. 1975, ApJ, 195, L93

Anders, E., \& Grevesse, N. 1989, Geochim. Cosmochim. Acta, 53, 197

Antia, H. M., \& Basu, S. 2005, ApJ, 620, L129

Arnaud, K. A. 1996, in Astronomical Data Analysis Software and Systems V, ed. G. H. Jacoby, \& J. Barnes, ASP Conf. Ser., 101, 17

Asplund, M., Grevesse, N., \& Sauval, A. J. 2005, in Cosmic Abundances as Records of Stellar Evolution and Nucleosynthesis, ed. T. G. Barnes, III, \& F. N. Bash, ASP Conf. Ser., 336, 25

Bahcall, J. N., Basu, S., Pinsonneault, M., \& Serenelli, A. M. 2005a, ApJ, 618, 1049

Bahcall, J. N., Basu, S., \& Serenelli, A. M. 2005b, ApJ, 631, 1281

Basu, S., \& Antia, H. M. 2004, ApJ, 606, L85

Dere, K. P., Landi, E., Mason, H. E., Monsignori Fossi, B. C., \& Young, P. R. 1997, A\&AS, 125, 149

Drake, J. J., \& Testa, P. 2005, Nature, 436, 525

Favata, F., Micela, G., Baliunas, S. L., et al. 2004, A\&A, 418, L13

Fruscione, et al. 2006, SPIE Proc., 6270

Geiss, J. 1998, Space Sci. Rev., 85, 241
Grevesse, N., \& Sauval, A. J. 1998, Space Sci. Rev., 85, 161

Güdel, M. 2004, A\&AR, 12, 71

Hempelmann, A., Robrade, J., Schmitt, J. H. M. M., et al. 2006, A\&A, 460, 261

Judge, P. G., Solomon, S. C., \& Ayres, T. R. 2003, ApJ, 593, 534

Landi, E., Del Zanna, G., Young, P. R., et al. 2006, ApJS, 162, 261

Liefke, C., \& Schmitt, J. H. M. M. 2006, A\&A, 458, L1

Loiseau, N., Ehle, M., Pollock, A., et al. 2006,

http://xmm.esac.esa.int/external/xmm_user_support/ documentation/sas_usg/USG/USG.html

McKenzie, D. L., \& Feldman, U. 1992, ApJ, 389, 764

McKenzie, J. F., Sukhorukova, G. V., \& Axford, W. I. 1998, A\&A, 332, 367

Ness, J.-U., \& Wichmann, R. 2002, Astron. Nachr., 323, 129

Peres, G., Orlando, S., Reale, F., Rosner, R., \& Hudson, H. 2000, ApJ, 528, 537

Raassen, A. J. J., Mewe, R., Audard, M., et al. 2002, A\&A, 389, 228

Raassen, A. J. J., Ness, J.-U., Mewe, R., et al. 2003, A\&A, 400, 671

Ramaty, R., Mandzhavidze, N., Kozlovsky, B., \& Murphy, R. J. 1995, ApJ, 455, L193

Robrade, J., Schmitt, J. H. M. M., \& Favata, F. 2005, A\&A, 442, 315

Robrade, J., Schmitt, J. H. M. M., \& Hempelmann, A. 2007, Mem. Soc. Astron. Ital., 78, 311

Sanz-Forcada, J., Favata, F., \& Micela, G. 2004, A\&A, 416, 281

Schmelz, J. T. 1993, ApJ, 408, 373

Schmelz, J. T., Nasraoui, K., Roames, J. K., Lippner, L. A., \& Garst, J. W. 2005, ApJ, 634, L197

Smith, R. K., Brickhouse, N. S., Liedahl, D. A., \& Raymond, J. C. 2001, ApJ, 556, L91

Telleschi, A., Güdel, M., Briggs, K., et al. 2005, ApJ, 622, 653

Turck-Chièze, S., Couvidat, S., Piau, L., et al. 2004, Phys. Rev. Lett., 93, 211102

von Steiger, R., \& Geiss, J. 1989, A\&A, 225, 222

Widing, K. G., \& Feldman, U. 1995, ApJ, 442, 446

Wood, B. E., \& Linsky, J. L. 2006, ApJ, 643, 444

Young, P. R. 2005, A\&A, 444, L45 